V3.3 MARCH 21, 2022

Preprint typeset using $\mathrm{LAT}_{\mathrm{E}} \mathrm{X}$ style emulateapj v. 5/2/11

\title{
DUST IN ACTIVE GALACTIC NUCLEI: ANOMALOUS SILICATE TO OPTICAL EXTINCTION RATIOS?
}

\author{
JiANWEI LYU ${ }^{1,2}$, LeI $\mathrm{HAO}^{1, \dagger}$, AND Aigen $\mathrm{LI}^{2}$ \\ v3.3 March 21, 2022
}

\begin{abstract}
Dust plays a central role in the unification theory of active galactic nuclei (AGNs). However, little is known about the nature (e.g., size, composition) of the dust that forms a torus around the AGN. In this Letter we report a systematic exploration of the optical extinction $\left(A_{V}\right)$ and the silicate absorption optical depth $\left(\Delta \tau_{9.7}\right)$ of 110 type 2 AGNs. We derive $A_{V}$ from the Balmer decrement based on Sloan Digital Sky Survey data, and $\Delta \tau_{9.7}$ from the Spitzer/InfraRed Spectrograph data. We find that with a mean ratio of $\left\langle A_{V} \mid \Delta \tau_{9.7}\right\rangle \lesssim 5.5$, the optical-to-silicate extinction ratios of these AGNs are substantially lower than that of the Galactic diffuse interstellar medium (ISM) for which $A_{V} / \Delta \tau_{9.7} \approx 18.5$. We argue that the anomalously low $A_{V} / \Delta \tau_{9.7}$ ratio could be due to the predominance of larger grains in the AGN torus compared to that in the Galactic diffuse ISM.
\end{abstract}

Subject headings: dust, extinction — galaxies: active — galaxies: ISM — infrared: galaxies

\section{INTRODUCTION}

Active galaxies are eye-catching due to the ongoing energetic accretion of nuclear material by their central supermassive black holes. Observations have shown that a large fraction of such activities are obscured by large columns of dust and gas. The unified model of active galactic nuclei (AGNs; see Antonucci 1993), which features a torus-shaped structure of obscuring material, successfully explains a large number of AGN observables. Nevertheless, the properties of the dust in AGNs remain poorly understood (see Li 2007).

An accurate knowledge of the dust extinction is crucial to recover the AGN intrinsic spectra, and it also influences the estimation of important parameters such as the AGN luminosity, black hole mass, and Eddington ratio. Numerous efforts have been made to expand such knowledge but have led to contradicting results: statistical studies on the reddening of quasars suggest a steeply rising extinction curve like (or even steeper than) that of the Small Magellanic Cloud (SMC), which indicates the predominance of small grains (Hall et al.|2002; Richards et al. 2003; Hopkins et al. 2004; Glikman et al. 2012; Jiang et al. 2013), while a flat or "gray" extinction curve (which varies little with wavelength) has also been proposed, which suggests the richness of large grains (Gaskell et al. 2004; Czernv et al. 2004; Gaskell \& Benker 2007). We note that in deriving these AGN extinction curves, one could suffer from the possible variations in the AGN intrinsic spectra, orientation effects, or potentially biased sampling (Czernv 2007).

Alternatively, one could also probe the dust size and composition through the dust infrared (IR) spectroscopy of AGNs. As shown in Li et al. (2008), Smith et al. (2010), and Köhler \& Li (2010), the $9.7 \mu \mathrm{m}$ silicate emission (Hao et al. 2005b; Siebenmorgen et al. 2005; Sturm et al. 2005) and absorption (Jaffe et al. 2004)

\footnotetext{
${ }^{1}$ Key Laboratory for Research in Galaxies and Cosmology, Shanghai Astronomical Observatory, Chinese Academy of Sciences, 80 Nandan Road, Shanghai 200030, China

2 Department of Physics and Astronomy, University of Missouri, Columbia, MO 65211, USA

† haol@shao.ac.cn
}

spectra of AGNs are diagnostic of the silicate composition and size.

Dust size could also be probed through $A_{V} / \Delta \tau_{9.7}$, the ratio of the visual extinction $A_{V}$ to the $9.7 \mu \mathrm{m}$ silicate absorption depth $\Delta \tau_{9.7}$ (Gao et al. 2010; Z. Shao et al. 2014 , in preparation): for compact, spherical silicate dust, $A_{V} / \Delta \tau_{9.7}$ peaks at a grain size of $a \sim 0.2 \mu \mathrm{m}$ and drops precipitously with the increase of the dust size. In the local interstellar medium (ISM) of the Milky Way, $A_{V} / \Delta \tau_{9.7} \approx 18.5 \pm 2$ (Roche \& Aitken 1984; see Table 1 of Draine 2003 for a summary). In this Letter we report a considerably lower $A_{V} / \Delta \tau_{9.7}$ ratio for 110 type 2 AGNs (Section 3), with $A_{V}$ determined from the Balmer decrement based on the Sloan Digital Sky Survey (SDSS) data, and $\Delta \tau_{9.7}$ from the $9.7 \mu \mathrm{m}$ Spitzer/InfraRed Spectroscope (IRS) absorption spectra (Section 2). The anomalously low $A_{V} / \Delta \tau_{9.7}$ ratio suggests the predominance of larger grains in the AGN torus compared to the typical size of $a \sim 0.1 \mu \mathrm{m}$ in the Galactic diffuse ISM (Section (4).

\section{DATA AND MEASUREMENTS}

\subsection{Sample}

We collect all type 2 AGNs from the $S^{3} A G A$ sample (Spitzer/IRS-SDSS Spectral Atlas of Galaxies and AGN; L. Hao et al. 2014, in preparation). $S^{3} A G A$ is a heterogeneous collection of galaxies that have Spitzer/IRS (Houck et al. 2004) low-resolution spectra and SDSS spectroscopic observations (Data Release 7; Abazajian et al. 2009) within a $3^{\prime \prime}$ searching radius. The whole $S^{3} A G A$ sample contains 139 type 1 AGNs, 114 type 2 AGNs, 241 star-forming (SF) galaxies, 103 AGN$\mathrm{SF}$ composites, and 1 quiescent galaxy. These classifications are made based on their SDSS optical spectra (see Hao et al. 2005a). Type 1 AGNs are those with broad $\mathrm{H} \alpha$ emission lines (with FWHM $>1200 \mathrm{~km} \mathrm{~s}^{-1}$ ). Type 2 AGNs are identified with the typical "Baldwin, Phillips \& Terlevich" diagram (Baldwin et al. 1981). This sample spans a redshift range of $z \sim 0.001-0.25$, corresponding to a physical size of $\sim 0.06-18 \mathrm{kpc}$ in the SDSS $3^{\prime \prime}$ aperture.

The low-resolution mid-IR spectra of Spitzer are adopted from the Cornell Atlas of Spitzer/IRS Sources 
(CASSIS; Lebouteiller et al. 2011). We use the CASSIS v4 data. In this version, the intermediate products of the Spitzer Science Center (SSC) pipeline release S18.7.0 are processed with the SMART software with a dedicated spectral extraction pipeline developed by the CASSIS team. We further combine the spectra of the Short-Low (SL) and Long-Low (LL) modules and scale the observed flux of LL to SL. Spikes and edge fringes are removed carefully. The final order-combined midIR spectra span a wavelength coverage of $5.2-38 \mu \mathrm{m}$ in the observer's frame, with a spectral resolution of $\sim 60$ 127 . We do not apply any aperture correction since the fiber size of SDSS $\left(3^{\prime \prime}\right)$ and slit width of Spitzer/IRS SL $\left(\sim 3^{\prime \prime} .6\right)$ are comparable.

\subsection{Extinction Determination}

The Balmer decrement can be used to trace the optical extinction: the difference between the observed and intrinsic ratios of two hydrogen recombination lines yields the amount of dust reddening. For the SDSS spectra, we subtract the stellar continuum, decompose the $[\mathrm{N} I \mathrm{II}]+\mathrm{H} \alpha$ region and $\mathrm{H} \beta$ region with multiple Gaussian components, and examine each fit by eye with the aid of the $\chi^{2}$ statistic.

We estimate the visual extinction from the measured $\mathrm{H} \alpha / \mathrm{H} \beta$ ratios, assuming a "screen" dust configuration:

$$
A_{V}=1.086 \times \xi \times \ln \left\{\frac{(\mathrm{H} \alpha / \mathrm{H} \beta)_{\mathrm{obs}}}{(\mathrm{H} \alpha / \mathrm{H} \beta)_{\mathrm{int}}}\right\},
$$

where

$$
\xi \equiv\left(A_{V} / A_{\mathrm{H} \alpha}\right) \times\left(A_{\mathrm{H} \beta} / A_{\mathrm{H} \alpha}-1\right)^{-1} .
$$

$A_{\mathrm{H} \alpha}$ and $A_{\mathrm{H} \beta}$ are the extinction at the $\mathrm{H} \alpha(6562 \AA)$ and $\mathrm{H} \beta$ (4831 $\AA$ ) bands, respectively. The parameter $\xi$ only depends on how the extinction varies with wavelength: the grayer an extinction curve is, the larger $\xi$ is. For a gray-type extinction curve such as Gaskell et al. (2004), $\xi \approx 3.61$ (see Table 11). We adopt a standard "Case B" recombination for the intrinsic Balmer decrement, i.e., $(\mathrm{H} \alpha / \mathrm{H} \beta)_{\text {int }} \approx 3.1$ (Osterbrock \& Ferland 2006).

We define the silicate strength as

$$
S_{9.7}=-\ln \left(\frac{I_{\lambda *, \mathrm{obs}}}{I_{\lambda *, \mathrm{cont}}}\right)
$$

where $\lambda *$ is the wavelength of the $9.7 \mu \mathrm{m}$ silicate feature peak, $I_{\lambda *, \text { obs }}$ and $I_{\lambda * \text { cont }}$ are the corresponding observed and continuum intensity, respectively. We estimate the silicate continuum using the interpolation method of Spoon et al. (2007) and the PAHFIT decomposition method (Smith et al. 2007). In general, the results based on these two methods agree well. The final adopted $9.7 \mu \mathrm{m}$ silicate strength is derived based on the interpolated continuum with the offset between these two approaches as the error (See L. Hao et al. 2014, in preparation for details).

We do not apply Galactic extinction correction on any spectra, since none of our objects locates along the Galactic plane. SDSS J031501.41+420208.9 is the closest to the Galactic plane with its Galactic latitude at $b=-13^{\circ} .3$.

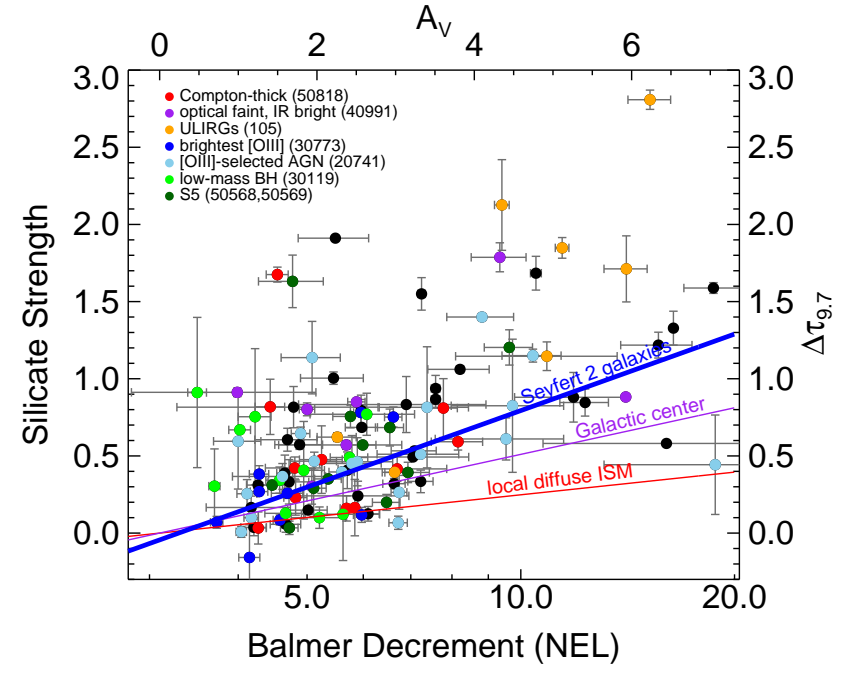

Fig. 1.- Observed Balmer decrements $(\mathrm{H} \alpha / \mathrm{H} \beta)_{\text {obs }}$ of the hydrogen narrow emission lines (NEL) vs. the $9.7 \mu \mathrm{m}$ silicate absorption strengths $S_{9.7}$ for our sample. Also shown are the optical extinction $A_{V}$ (upper axis) derived from Equation (1) and the $9.7 \mu \mathrm{m}$ silicate absorption optical depth $\Delta \tau_{9.7}$ (right axis) derived from Equation (4), assuming a "screen" dust geometry (i.e., $\Delta \tau_{9.7}=S_{9.7}$ ). Some of the data points obtained from different Spitzer programs are coded with different colors in order to show if the sources from any single program dominate or cluster in the figure (with program features and IDs shown in the legend). The thick blue line is a linear fit of $A_{V} / \Delta \tau_{9.7} \approx 5.5 \pm 2.7$ (with an additional $y$-axis intercept $\sim-0.04 \pm 0.31$ and a reduced $\left.\chi^{2} \approx 43.2\right)$. The red and purple solid lines plot $A_{V} / \Delta \tau_{9.7} \approx 18.5$ for the local ISM of the Milky Way, and $A_{V} / \Delta \tau_{9.7} \approx 9$ for the Galactic center, resectively.

\section{RESULTS: ANOMALOUSLY LOW $A_{V} / \Delta \tau_{9.7}$ RATIOS IN TYPE 2 AGNS}

For a "screen" dust configuration, $\Delta \tau_{9.7}=S_{9.7}$. Therefore, one would expect a relation between the silicate absorption strength $S_{9.7}$ and the Balmer decrement:

$S_{9.7} \approx 1.086 \times \xi \times\left(A_{V} / \Delta \tau_{9.7}\right)^{-1} \times \ln \left\{\frac{(\mathrm{H} \alpha / \mathrm{H} \beta)_{\text {obs }}}{(\mathrm{H} \alpha / \mathrm{H} \beta)_{\mathrm{int}}}\right\}$.

Figure 1 shows the distribution of this sample on a plot of the observed Balmer decrement $(\mathrm{H} \alpha / \mathrm{H} \beta)_{\text {obs }}$ versus the observed silicate strength $S_{9.7}$. A linear relation between $S_{9.7}$ and $\ln (\mathrm{H} \alpha / \mathrm{H} \beta)_{\text {obs }}$ is clearly seen (albeit with a large scatter). The slope of the correlation is $1.086 \times \xi \times\left(A_{V} / \Delta \tau_{9.7}\right)^{-1}$. For an assumed dust configuration, this slope is uniquely determined by the observed $S_{9.7}$ and $\ln (\mathrm{H} \alpha / \mathrm{H} \beta)_{\text {obs }}$. Hence, for a given extinction law, $A_{V} / \Delta \tau_{9.7} \propto \xi$.

Guided by Equation (4), we perform a linear weighted least-squares fit to the observationally determined parameters $S_{9.7}$ and $\ln (\mathrm{H} \alpha / \mathrm{H} \beta)_{\text {obs }}$ shown in Figure 1 . The Spearman correlation test gives a coefficient $r \approx 0.53$ with significance $p \approx 2.4 \times 10^{-9}$, suggesting a moderate correlation between $S_{9.7}$ and $\ln (\mathrm{H} \alpha / \mathrm{H} \beta)_{\text {obs }}$. Assuming a Gaskell et al.-type extinction curve Gaskell et al. $2004)$ for which $\xi \approx 3.61$, we obtain $A_{V} / \Delta \tau_{9.7} \approx 5.5$. The bootstrap method is used to estimate the errors of the fitted parameters with 5000 realizations (sampling with replacement), and the resulting $1 \sigma$ uncertainty for $A_{V} / \Delta \tau_{9.7}$ is 2.4 . In the fitting, we allow a small value of additional intercept to account for the $(\mathrm{H} \alpha / \mathrm{H} \beta)_{\text {int }}$ term 
and the possible measurement uncertainties. The fitted additional intercept of $\sim-0.04 \pm 0.31$ is indeed small.

The $A_{V} / \Delta \tau_{9.7} \approx 5.5 \pm 2.7$ ratio obtained above is significantly lower than that of the Galactic diffuse ISM $\left(A_{V} / \Delta \tau_{9.7} \approx 18.5 \pm 2\right.$, see review by Draine 2003). As shown in Figure 2 and Table 1 the Gaskell et al. (2004) extinction curve is the grayest and corresponds to the largest $\xi$ among all the extinction laws commonly considered in AGN studies. As a result, any other extinction curve would give an even lower $A_{V} / \Delta \tau_{9.7}$.

The scatter of the correlation shown in Figure1 is large (with a reduced $\chi^{2} \approx 43.2$ ). This is understandable since the intrinsic properties of the dusty structures may be very different among type 2 AGNs. It is also possible that there exists a systematic secondary effect in the correlation causing the scatter (see discussion in Section 4). Nevertheless, it is clear, as can be seen from Figure 1 . that most type 2 AGNs lie above the lines given by the Galactic $A_{V} / \Delta \tau_{9.7}$ ratios.

In our fitting, we exclude 4 out of the 114 type 2 AGNs in the $S^{3} A G A$ sample: 3 of them have the Balmer decrement signal-to-noise ratio smaller than 3.0 and 1 source shows $(\mathrm{H} \alpha / \mathrm{H} \beta)_{\mathrm{obs}}<3.1$. Sample selection is unlikely to bring significant bias on our result. Our 110 AGNs are selected from 29 Spitzer programs whose scientific goals are substantially different, without a single program dominating the sources or clustering in Figure 1 (see the color-coded data points).

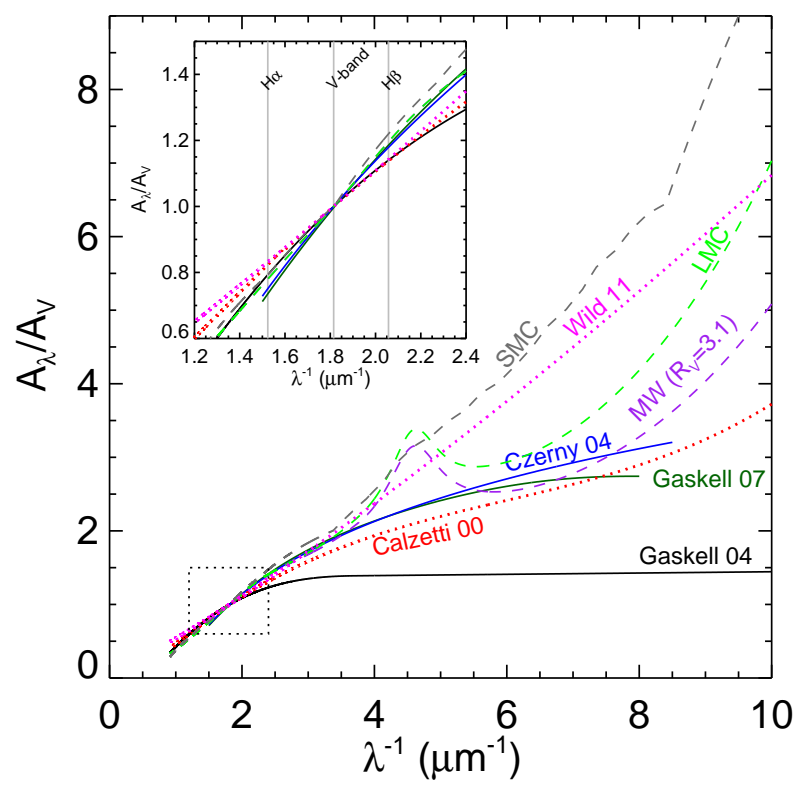

FIG. 2.- Extinction curves commonly considered in AGN studies: flat or gray curves of Gaskell et al. (2004), Czerny et al. (2004), and Gaskell \& Benker (2007). Also shown are the extinction curves of the Milky Way diffuse ISM $\left(R_{V}=3.1\right)$, the LMC, the SMC, and the steep curves of Wild et al. (2011). The attenuation curve of starburst galaxies of Calzetti et al. (2000) is also plotted. The insert panel highlights the extinction curves in the $\mathrm{H} \alpha, V$, and $\mathrm{H} \beta$ bands.

\section{DISCUSSION}

The "screen" dust configuration is a simple assumption for the obscuring AGN dust structure. To examine the
TABLE 1

$A_{V} / \Delta \tau_{9.7}$ Derived from Various Extinction Curves

\begin{tabular}{lcccc}
\hline \hline \multicolumn{1}{c}{ Extinction Law } & $A_{\mathrm{H} \beta} / A_{\mathrm{H} \alpha}$ & $A_{V} / A_{\mathrm{H} \alpha}$ & $\xi$ & $A_{V} / \Delta \tau_{9.7}$ \\
\hline Gaskell et al. (2004) & 1.34 & 1.21 & 3.61 & 5.5 \\
Gaskell \& Benker (2007) & 1.61 & 1.37 & 2.25 & 3.4 \\
Czerny et al. (2004) & 1.60 & 1.37 & 2.27 & 3.5 \\
\hline MW $R_{V}=3.1$ & 1.51 & 1.29 & 2.44 & 3.7 \\
LMC Average & 1.53 & 1.23 & 2.30 & 3.5 \\
SMC & 1.54 & 1.28 & 2.38 & 3.6 \\
\hline Wild et al. (2011) & 1.37 & 1.20 & 3.22 & 4.9 \\
Calzetti et al. (2000) & 1.53 & 1.30 & 2.44 & 3.7 \\
\hline
\end{tabular}

Note. $-A_{V} / \Delta \tau_{9.7} \propto \xi$ where $\xi \equiv\left(A_{V} / A_{\mathrm{H} \alpha}\right) /\left(A_{\mathrm{H} \beta} / A_{\mathrm{H} \alpha}-1\right)$.

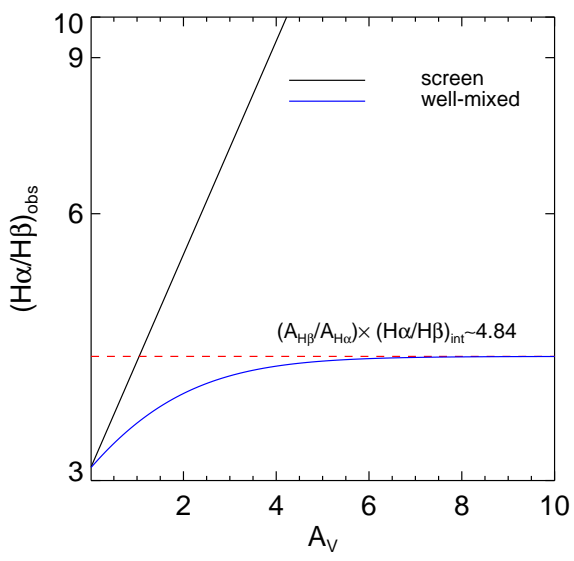

FIG. 3.- Optical extinction $A_{V}$ derived from the observed Balmer decrement $(\mathrm{H} \alpha / \mathrm{H} \beta)_{\text {obs }}$ with the assumption of the gray extinction curve of Gaskell et al. (2004) in combination with a "screen" (black solid line) or "well-mixed" (blue solid line) dust configuration. Also shown is the asymptotic value of $(\mathrm{H} \alpha / \mathrm{H} \beta)_{\mathrm{obs}} \approx\left(A_{\mathrm{H} \beta} / A_{\mathrm{H} \alpha}\right) \times(\mathrm{H} \alpha / \mathrm{H} \beta)_{\mathrm{int}} \approx 4.84$ for the "screen" geometry at $A_{V} \rightarrow \infty$ (red dashed line; see Equation (5)).

possible effects caused by the deviation from a "screen" configuration, we consider two cases: (1) one is an extreme case in which the dust is assumed to thoroughly mix with the light source, but the dust distribution is still "smooth," and (2) one is a clumpy distribution of discrete dust clouds (e.g., see Nenkova et al. 2008a,b; Nikutta et al. 2009).

In a "well-mixed" geometry, the observed intensity $I_{\lambda}$ relates to the intensity of the source $I_{\lambda}^{\mathrm{o}}$ through $I_{\lambda}=$ $I_{\lambda}^{\mathrm{o}}\left\{1-\exp \left(-\tau_{\lambda}\right)\right\} / \tau_{\lambda}$, where $\tau_{\lambda}$ is the optical depth $\left(\tau_{\lambda}=\right.$ $\left.A_{\lambda} / 1.086\right)$ at wavelength $\lambda$ (Mathis 1972). Therefore, the visual extinction can be deduced from the observed Balmer decrement through

$\frac{(\mathrm{H} \alpha / \mathrm{H} \beta)_{\mathrm{obs}}}{(\mathrm{H} \alpha / \mathrm{H} \beta)_{\mathrm{int}}}=\left(\frac{A_{\mathrm{H} \beta}}{A_{\mathrm{H} \alpha}}\right) \times \frac{1-\exp \left\{-\left(A_{V} / 1.086\right)\left(A_{\mathrm{H} \alpha} / A_{V}\right)\right\}}{1-\exp \left\{-\left(A_{V} / 1.086\right)\left(A_{\mathrm{H} \beta} / A_{V}\right)\right\}}$.

As shown in Figure 3, for a given extinction curve (which specifies $A_{\mathrm{H} \beta} / A_{\mathrm{H} \alpha}, A_{\mathrm{H} \alpha} / A_{V}$, and $A_{\mathrm{H} \beta} / A_{V}$ ), the "mixed" dust geometry always requires a larger optical extinction $A_{V}$ to account for the same Balmer decrement $(\mathrm{H} \alpha / \mathrm{H} \beta)_{\text {obs }}$. In this case, we will derive a higher $A_{V} / \Delta \tau_{9.7}$ compared to the "screen" scenario for the same Balmer decrement and silicate data. Meanwhile, as can be seen in Equation (5) and in Figure 3 the observed Balmer decrement cannot exceed $\left(A_{\mathrm{H} \beta} / A_{\mathrm{H} \alpha}\right) \times$ 
$(\mathrm{H} \alpha / \mathrm{H} \beta)_{\text {int }} \approx 5.0$ (corresponding to $A_{\mathrm{H} \beta} / A_{\mathrm{H} \alpha} \approx 1.61$ for the extinction curve of Gaskell \& Benker 2007), for any reasonable extinction curves considered in Figure 2 and in Table 1. However, as shown in Figure [1] a large fraction of the sources have $(\mathrm{H} \alpha / \mathrm{H} \beta)_{\mathrm{obs}}>5$ and they dominate the overall trend of $A_{V} / \Delta \tau_{9.7}$. Therefore, the obscuring dust can not be in a "well-mixed" geometry. For the $9.7 \mu \mathrm{m}$ silicate optical depth, one would expect some degree of mixing since the silicate dust in the warm inner torus will emit at $9.7 \mu \mathrm{m}$. Taking into account the silicate emission, one should derive a higher $\Delta \tau_{9.7}$ and hence an even lower $A_{V} / \Delta \tau_{9.7}$.

For a clumpy torus, we assume a Poisson distribution of discrete clouds. Let $N$ be the average number of clouds along a radial equatorial line of sight, and let $\tau_{\lambda, c}$ be the optical depth of a single cloud at wavelength $\lambda$. The observed intensity becomes $I_{\lambda}=I_{\lambda}^{\mathrm{o}} \exp \left\{-N\left[1-\exp \left(-\tau_{\lambda, c}\right)\right]\right\}$. For the "screen" configuration, we can consider the "screen" as a smooth, continuous distribution of $N$ clouds. Hence the optical extinction and silicate optical depth are $A_{V}=1.086 N \tau_{V, c}$ and $\Delta \tau_{9.7}=N \Delta \tau_{9.7, c}$, respectively. Consequently, the ratio of the clumpy $\left(A_{V} / \Delta \tau_{9.7}\right)_{\text {clum }}$ to the screen $\left(A_{V} / \Delta \tau_{9.7}\right)_{\mathrm{scrn}}$ becomes $\left(A_{V} / \Delta \tau_{9.7}\right)_{\text {clum }} /\left(A_{V} / \Delta \tau_{9.7}\right)_{\text {scrn }}=$ $\left\{1-\exp \left(-\tau_{V, c}\right)\right\} /\left\{1-\exp \left(-\tau_{9.7, c}\right)\right\} \times\left\{\tau_{9.7, c} / \tau_{V, c}\right\}$. It is easy to show $\left(A_{V} / \Delta \tau_{9.7}\right)_{\text {clum }}<\left(A_{V} / \Delta \tau_{9.7}\right)_{\text {scrn }}$. Therefore, the low optical-to-silicate extinction ratio of $A_{V} / \Delta \tau_{9.7} \approx 5.5$ derived in Section 3 indeed seems to already be in the high end. Nevertheless, we note that in reality, the actual optical depth of a clumpy geometry can be much more complicated than assumed above. Besides, a clumpy geometry cannot produce silicate depths greater than 0.5 (e.g., see Levenson et al. 2007; Nikutta et al. 2009), while a number of $S^{3} A G A$ AGNs have silicate absorption $S_{9.7}>0.5$. Thus, detailed modeling is needed to fully address this issue.

Dust scatters and absorbs light most effectively when its size $a$ is comparable to the wavelength $\lambda$ of the light (i.e., $2 \pi a / \lambda \sim 1$ ). For the optical light, grains of $a \sim 0.1 \mu \mathrm{m}$ are an effective scatter and absorber. For larger grains (say, $a \gtrsim 0.5 \mu \mathrm{m}$ ), they are less effective in extinguishing the optical light but become effective in producing the $9.7 \mu \mathrm{m}$ silicate absorption feature (see Z . Shao et al. 2014, in preparation). The low $A_{V} / \Delta \tau_{9.7}$ ratio of AGNs could readily be explained in terms of dust larger than the submicro-sized interstellar dust. In Figure 4. we show the optical-to-silicate extinction ratios $\left(A_{V} / \Delta \tau_{9.7}\right)$ as a function of grain radii $a$ for compact, spherical silicate grains calculated from Mie theory and the dielectric functions of "astronomical silicates" from Draine \& Lee (1984). It is seen that $A_{V} / \Delta \tau_{9.7}$ peaks at $a \sim 0.2 \mu \mathrm{m}$ and rapidly decreases with the increase of $a$. At $a>0.44 \mu \mathrm{m}$, we see $A_{V} / \Delta \tau_{9.7}<5.5$. Meanwhile, as shown in Figure 4 , one could also achieve $A_{V} / \Delta \tau_{9.7}<5.5$ with $a<0.09 \mu \mathrm{m}$. However, it is unlikely for these small grains to survive in the hostile AGN circumnuclear environments, and the spectroscopic studies of the $9.7 \mu \mathrm{m}$ silicate feature of AGNs all point to dust much larger than 0.1 $\mu \mathrm{m}$ (see Li et al. 2008; Köhler \& Li 2010; Smith et al. 2010). We note that the observed high end of $A_{V} / \Delta \tau_{9.7} \approx 5.5$ does not necessarily imply that the AGN dust grains are all larger than $a \approx 0.44 \mu \mathrm{m}$, since the optical extinction is not exclusively contributed by silicate dust, although the fractional contribution of carbon dust to $A_{V}$ is not known.

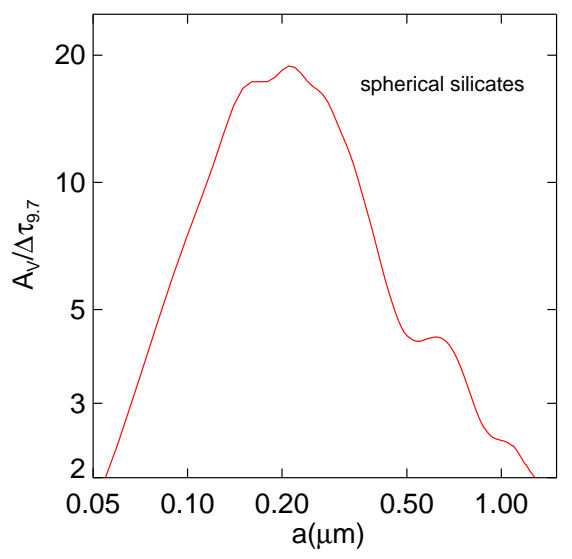

FIG. 4.- Optical to silicate extinction ratio $\left(A_{V} / \Delta \tau_{9.7}\right)$ of spherical silicate dust as a function of dust size.

There is evidence that suggests that the dust in the AGN torus is larger than the dust in the ISM. The gray extinction derived for AGNs implies that the size distribution of the dust in AGNs is skewed toward substantially large grains (see Section 1). Some infrared interferometry studies also show various indications that the grain size around the torus might be larger (Kishimoto et al. 2007, 2009; Hönig et al. 2013; Burtscher et al. 2013). Maiolino et al. (2001b) found that, for 16 of the 19 AGNs they studied, their $E(B-$ $V) / N_{\mathrm{H}}$ and $A_{V} / N_{\mathrm{H}}$ are significantly lower than the Galactic standard values. Maiolino et al. (2001a) ascribed these reduced ratios in AGNs (often with a solar or higher metallicity) to grain growth through coagulation in the dense circumnuclear regions. In this case, we could expect a dust size distribution skewed toward large grains, leading a flat extinction curve (but see Weingartner \& Murray 2002, , who argued that the $\mathrm{X}$-ray absorption and optical extinction may occur in distinct media). We note that the preferential destruction of small dust by X-ray photons in the AGN torus could also lead to the predominance of large dust and result in reduced $E(B-V) / N_{\mathrm{H}}$ and $A_{V} / N_{\mathrm{H}}$ ratios.

A low $A_{V} / \Delta \tau_{9.7}$ ratio could also be caused by a smaller abundance of carbonaceous dust in AGNs than in the ISM. Toward the Galactic center, the optical-to-silicate extinction ratio is $A_{V} / \Delta \tau_{9.7} \approx 9$ (Roche \& Aitken 1985) which is just $\sim 1 / 2$ of that of the local ISM. Roche \& Aitken (1985) attributed this to the relative abundance of carbonaceous dust to silicate dust: in the Galactic center there are more oxygen-rich stars which make silicate dust (but see Gao et al. 2010). In AGN torus, if carbon dust is preferentially destroyed, one would also expect a low $A_{V} / \Delta \tau_{9.7}$ ratio since carbon dust only contributes to $A_{V}$ while silicate dust contributes to both $A_{V}$ and $\Delta \tau_{9.7}$. The harsh radiation in AGNs is expected to destroy dust. However, Perna et al. (2003) found that, subject to X-ray/UV radiation, silicate grains would be preferentially destroyed with respect to graphite. 
Leipski et al. (2007) derived $A_{V} \approx 2.4$ (from the narrow line Balmer decrement) and silicate optical depth $\Delta \tau_{9.7} \approx 0.34$ for a type 2 AGN J12324114+1112587. This also implies a low optical-to-silicate extinction ratio of $A_{V} / \Delta \tau_{9.7} \approx 7.1$ compared with the Galactic value. Leipski et al. (2007) explained this as that the optical extinction and the silicate absorption may take place at different regions: the silicate absorption mainly arises from the dust which is concentrated toward the center of the galaxy, while the NLR emission comes from larger scales and is additionally absorbed by ambient dust in the host galaxy. Goulding et al. (2012) explored the origin of the $9.7 \mu \mathrm{m}$ silicate absorption in 20 nearby $(z<0.05)$ Compton-thick AGNs. They accurately measured the silicate absorption in archival low-resolution Spitzer/IRS spectroscopy. They found that $\sim 45 \%$ of the sources have strong silicate absorption with $S_{9.7}>0.5$. Differing from Leipski et al. (2007), Goulding et al. (2012) argued that the dominant contribution to the observed silicate absorption is made by the dust located in the host galaxy and not necessarily in a compact obscuring torus surrounding the central engine. We note that the discussions on the low $A_{V} / \Delta \tau_{9.7}$ ratio presented in this work are based on the assumption that both the optical extinction and the silicate absorption are from the torus. The optical extinction derived from the NLR Balmer decrement may not be fully representative of the torus extinction since the NLR may not be obscured by the entire torus. The extinction can also come from the dust in hosts. However, if the hosts also contribute to the midIR extinction, a low value of $A_{V} / \Delta \tau_{9.7}$ ratio in AGNs compared to the Galactic ISM can still be expected, since $\left[A_{V}\right.$ (host) $+A_{V}$ (torus) $] /\left[\Delta \tau_{9.7}\right.$ (host) $+\Delta \tau_{9.7}$ (torus) $]>$
$A_{V}$ (torus) $/ \Delta \tau_{9.7}$ (torus). We call on further studies on the origin of the optical and silicate extinction. Nevertheless, if the optical extinction and the silicate absorption are indeed from very different regions, one should not expect any correlation between $A_{V}$ and $\Delta \tau_{9.7}$. Mismatches in the extinction regions traced by optical and silicate absorption may act as the secondary effect, at most, in the correlation shown in Figure 1.

\section{SUMMARY}

We have determined $A_{V} / \Delta \tau_{9.7}$, the ratio of the visual extinction to the $9.7 \mu \mathrm{m}$ silicate absorption optical depth, of 110 type 2 AGNs. The mean ratio of $A_{V} / \Delta \tau_{9.7} \approx 5.5$ is considerably lower than that of the local ISM of the Milky Way. We attribute this anomalously low $A_{V} / \Delta \tau_{9.7}$ ratio of AGNs to a dust size distribution skewed toward substantially large grains, as a result of preferential destruction of small grains by X-ray/UV photons and/or grain growth in the dense circumnuclear regions of AGNs.

We thank J. Gao, B.W. Jiang, Z. Shao, Y. Xie and the anonymous referee for helpful suggestions. J.L. and L.H. are partially supported by the 973 Program of China (2013CB834905, 2009CB824800), the Strategic Priority Research Program "The Emergence of Cosmological Structures" of Chinese Academy of Sciences (XDB09030200), the Shanghai Pujiang Talents Program (10pj1411800) and NSFC 11073040. A.L. is supported in part by NSF AST-1311804 and NASA NNX14AF68G. The Cornell Atlas of Spitzer/IRS Sources (CASSIS) is a product of the Infrared Science Center at Cornell University, supported by NASA and JPL.

\section{REFERENCES}

Abazajian, K. N., Adelman-McCarthy, J. K., Agüeros, M. A., et al. 2009, ApJS, 182, 543

Antonucci, R. 1993, ARA\&A, 31, 473

Baldwin, J. A., Phillips, M. M., \& Terlevich, R. 1981, PASP, 93, 5 Burtscher, L., Meisenheimer, K., Tristram, K. R. W., et al. 2013, A\&A, 558, A149

Calzetti, D., Armus, L., Bohlin, R. C., et al. 2000, ApJ, 533, 682

Czerny, B. 2007, in ASP Conf. Ser. 373, The Central Engine of Active Galactic Nuclei, ed. L. C. Ho \& J.-W. Wang (San Francisco, CA: ASP), 586

Czerny, B., Li, J., Loska, Z., \& Szczerba, R. 2004, MNRAS, 348, L54

Draine, B. T. 2003, ARA\&A, 41, 241

Draine, B. T., \& Lee, H. M. 1984, ApJ, 285, 89

Gao, J., Jiang, B. W., \& Li, A. 2010, EP\&S, 62, 63

Gaskell, C. M., \& Benker, A. J. 2007, arXiv:0711.1013

Gaskell, C. M., Goosmann, R. W., Antonucci, R. R. J., \& Whysong, D. H. 2004, ApJ, 616, 147

Glikman, E., Urrutia, T., Lacy, M., et al. 2012, ApJ, 757, 51

Goulding, A. D., Alexander, D. M., Bauer, F. E., et al. 2012 ApJ, 755, 5

Hall, P. B., Anderson, S. F., Strauss, M. A., et al. 2002, ApJS, 141,267

Hao, L., Strauss, M. A., Tremonti, C. A., et al. 2005a, AJ, 129, 1783

Hao, L., Spoon, H. W. W., Sloan, G. C., et al. 2005b, ApJ, 625, L75

Hönig, S. F., Kishimoto, M., Tristram, K. R. W., et al. 2013 ApJ, 771, 87

Hopkins, P. F., Strauss, M. A., Hall, P. B., et al. 2004, AJ, 128, 1112

Houck, J. R., Roellig, T. L., van Cleve, J., et al. 2004, ApJS, 154, 18

Jaffe, W., Meisenheimer, K., Röttgering, H. J. A., et al. 2004, Nature, 429, 47

Jiang, P., Zhou, H., Ji, T., et al. 2013, AJ, 145, 157
Kishimoto, M., Hönig, S. F., Antonucci, R., et al. 2009, A\&A, 507, L57

Kishimoto, M., Hönig, S. F., Beckert, T., \& Weigelt, G. 2007, A\&A, 476, 713

Köhler, M., \& Li, A. 2010, MNRAS, 406, L6

Lebouteiller, V., Barry, D. J., Spoon, H. W. W., et al. 2011, ApJS, 196, 8

Leipski, C., Haas, M., Meusinger, H., et al. 2007, A\&A, 464, 895

Levenson, N. A., Sirocky, M. M., Hao, L., et al. 2007, ApJ, 654, L45

Li, A. 2007, in ASP Conf. Ser. 373, The Central Engine of Active Galactic Nuclei, ed. L. C. Ho \& J.-W. Wang (San Francisco, CA: ASP, 561

Li, M. P., Shi, Q. J., \& Li, A. 2008, MNRAS, 391, L49

Maiolino, R., Marconi, A., \& Oliva, E. 2001a, A\&A, 365, 37

Maiolino, R., Marconi, A., Salvati, M., et al. 2001b, A\&A, 365, 28

Mathis, J. S. 1972, ApJ, 176, 651

Nenkova, M., Sirocky, M. M., Ivezić, Ž., \& Elitzur, M. 2008a, ApJ, 685, 147

Nenkova, M., Sirocky, M. M., Nikutta, R., Ivezić, Ž., \& Elitzur, M. 2008b, ApJ, 685, 160

Nikutta, R., Elitzur, M., \& Lacy, M. 2009, ApJ, 707, 1550

Osterbrock, D. E., \& Ferland, G. J. 2006, Astrophysics of Gaseous Nebulae and Active Galactic Nuclei (2nd ed.; Sausalito, CA: University Science Books)

Perna, R., Lazzati, D., \& Fiore, F. 2003, ApJ, 585, 775

Richards, G. T., Hall, P. B., Vanden Berk, D. E., et al. 2003, AJ, 126, 1131

Roche, P. F., \& Aitken, D. K. 1984, MNRAS, 208, 481

-. 1985, MNRAS, 215, 425

Siebenmorgen, R., Haas, M., Krügel, E., \& Schulz, B. 2005, A\&A, 436, L5

Smith, H. A., Li, A., Li, M. P., et al. 2010, ApJ, 716, 490

Smith, J. D. T., Draine, B. T., Dale, D. A., et al. 2007, ApJ, 656, 770

Spoon, H. W. W., Marshall, J. A., Houck, J. R., et al. 2007, ApJ, $654, \mathrm{~L} 49$ 
Sturm, E., Schweitzer, M., Lutz, D., et al. 2005, ApJ, 629, L21

Weingartner, J. C., \& Murray, N. 2002, ApJ, 580, 88

Wild, V., Groves, B., Heckman, T., et al. 2011, MNRAS, 410, 1593 